EPJ manuscript No.

(will be inserted by the editor)

\title{
Searching for L-Violating Supersymmetry at the LHC
}

\author{
P. Z. Skands ${ }^{\text {a }}$ \\ Niels Bohr Institute, Blegdamsvej 17, 2100 Copenhagen $\varnothing$, Denmark
}

LU TP 01-32

\begin{abstract}
The possibility to simulate lepton number violating supersymmetric models has been introduced into the recently updated PYTHIA event generator, now containing 1278 decay channels of SUSY particles into SM particles via lepton number violating interactions. This generator has been used in combination with the ATLFAST detector simulation to study the impact of lepton number violation $(\not L)$ on event topologies in the ATLAS detector, and trigger menus designed for $\not /$-SUSY are proposed based on very general considerations. In addition, a rather preliminary analysis is presented on the possibility for ATLAS to observe a signal above the background in several mSUGRA scenarios, using a combination of primitive cuts and neural networks to optimize the discriminating power between signal and background events over regions of parameter space rather than at individual points. It is found that a $5 \sigma$ discovery is possible roughly for $m_{1 / 2}<1 \mathrm{TeV}$ and $m_{0}<2 \mathrm{TeV}$ with an integrated luminosity of $30 \mathrm{fb}^{-1}$, corresponding to one year of data taking with the LHC running at "mid-luminosity", $\mathcal{L}=3 \times 10^{33} \mathrm{~cm}^{-2} \mathrm{~s}^{-1}$.

PACS. 12.60.Jv Supersymmetric Models - 11.30.Fs Global symmetries - 13.85.-t Hadron-induced highand super-high-energy interactions
\end{abstract}

\section{Introduction}

Among the primary physics goals of the Large Hadron Collider at CERN, scheduled to turn on some time during 2006, are the exploration of the SM Higgs and top quark sectors, and the direct exploration of the $\mathrm{TeV}$ scale with the emphasis on Supersymmetry.

The motivations for believing that Supersymmetry is indeed a property of Nature are many, most importantly the natural and exhaustive extension of the Poincaré group furnished by the Supersymmetric operators [1], the possibility for exact unification of the SM gauge couplings as required by GUTs, the requirement of Supersymmetry for anomaly-cancellation in string theories, and perhaps most importantly, its providing a natural and elegant solution to the problem of scale hierarchy in the SM.

For this latter problem to be solved without unnatural finetuning order by order in perturbation theory, the sparticle masses must lie at or below the $\mathrm{TeV}$ scale, and so there is ample reason to believe that SUSY should be observable at the LHC (see e.g. [2]).

The potential for hadron colliders to observe Supersymmetry has been studied in great detail (see e.g. [3,

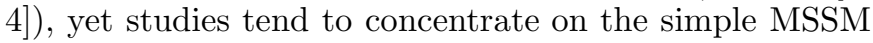
framework where both baryon and lepton number conservation are imposed. In section 2 we summarize the theoretical situation, pointing out that it is by no means obvious

\footnotetext{
a Now at Department of Theoretical Physics, Lund University, Sölvegatan 14A, S-22362 Lund (zeiler@thep.lu.se)
}

that baryon and lepton number should be conserved in supersymmetric theories.

The possibility to study lepton and baryon number violating Supersymmetry has already been included [5] in the Herwig event generator [6] and in SusyGen [7, and several studies exist for run II of the Tevatron (for reviews, see [8, 9], latest experimental results 10]) and to a lesser extent for the LHC (see e.g. [11, 12, 13, 14).

However, it has not before been possible to study the full range of decays in these supersymmetric models using the PyтhiA framework (and thus the string fragmentation model), and no dedicated LHC studies have been performed regarding triggers and sensitivity for these theories beyond cases where the LSP was simply forced to decay. In this paper, we go to the general case where all sparticles (excepting the gluino) are allowed to decay via lepton number violating couplings, introducing 1278 lepton number violating decay channels of sparticles to particles in the PYтHIA event generator.

For the purpose of studying the experimental signatures arising from the lepton number violating decay channels, and to quantify to some extent the "discovery potential" of the LHC, some selection of benchmark points is needed. 5 mSUGRA points and, for each of these, 9 scenarios for the 36 स coupling strengths are presented and discussed in section 3 .

From this point on, the analysis relies on technical aspects of the detector design (or rather, their representation in crude simulation algorithms), and so we are forced to distinguish between the ATLAS and CMS experiments. 
P. Z. Skands: Searching for L-Violating Supersymmetry at the LHC

Assuming to a first approximation that these experiments will have similar capabilities, we choose to concentrate on ATLAS in the remainder of the paper.

A first step is to define the data sample inside which IL-SUSY should be searched for. To this purpose, a selection of triggers designed for $\not L$-SUSY scenarios are proposed for "mid-luminosity" running of the $\operatorname{LHC}(\mathcal{L}=$ $\left.3 \times 10^{33} \mathrm{~cm}^{-2} \mathrm{~s}^{-1}\right)$ in section 0 .

Before going further, we note that the conventional procedure of studying a few benchmark points in detail is insufficient in the enlarged parameter space opened up by the $L$-violating couplings, even more so since the presence of such couplings also remove important cosmological contraints on the mSUGRA parameters. In particular, the Lightest Supersymmetric Particle (LSP) is no longer required to be neutral [15], and essentially no bound on the mSUGRA parameters can be obtained by requiring a relic density less than or equal to the density of dark matter in the universe.

In section 5, we make a first attempt at coming to grips with the size of the parameter space, proposing a method which relies on neural networks and the grouping of individual scenarios into classes, whereby regions rather than individual points become the studied objects in parameter space. In this work, we define just 2 classes of $L$ SUSY models, each containing 15 individual scenarios and a class consisting of 5 MSSM scenarios for reference. For each class, a neural network is trained with post-trigger events from all the scenarios in the class against background events, allowing the network to pick out general qualities common to each class without over-fitting to a particular model. Although we study only a few scenarios here, the usefulness of this method should become apparent when considering the requirements posed by more comprehensive scans over parameter space.

After some remarks pertaining to the dangers of using neural networks, we present results for the estimated ATLAS sensitivity, $S / \sqrt{S+B}$, for all scenarios with an assumed integrated luminosity of $30 \mathrm{fb}^{-1}$. The subsequent step of pinning down the model parameters once a signal has been observed, is clearly more model dependent and is not covered here. An up-to-date review can be found in [9].

A brief outlook and concluding remarks are given in section 6 .

\section{L-Violating SUSY}

In this part of the paper, we do not present new results. Rather, we summarize some theoretical considerations concerning $R$-violation which, although well documented in the literature, may not be in the active memory of all readers.

Despite the many attractive features of Supersymmetry, most notably that it provides a natural solution to the hierarchy problem, it is well known that the most general supersymmetric Lagrangian (containing all terms obeying $S U(3)_{C} \times S U(2)_{L} \times U(1)_{Y}$ gauge invariance and Supersymmetry) is utterly incompatible with experiment, re- gardless of whether the assumption of minimal particle content is made or not.

The reason is that baryon and lepton number conservation is not guaranteed by any of the symmetries just mentioned, and the accidental conservation of these quantum numbers in the SM does not hold when extending the SM with Supersymmetry; the full SUSY Lagrangian contains renormalizable lepton and baryon number violating operators 16] which are suppressed only by the SUSY breaking mass scale squared. Unless a much more powerful suppression mechanism is also at work, these operators result in a lifetime for the proton which would be measured in fractions of a second, to be compared with the experimental bound $\tau_{p}>1.6 \times 10^{33} \mathrm{yr}$ (at $90 \% \mathrm{CL}$ ) obtained by Super-Kamiokande in the $p \rightarrow e^{+} \pi^{0}$ channel [17].

The possibility that the couplings responsible for proton decay are just naturally small but not zero is almost out of the question. Assuming $M_{S U S Y} \approx 1 \mathrm{TeV}$, the product of the $\not B$ and $\not /$ couplings involved in proton decay is required to be less than $10^{-25}$ [18]. Since at least one of the couplings is then forced to have a value below $10^{-12}$ without any obvious suppression mechanism at work, it is more natural to assume that there is some additional symmetry in the theory, giving zero couplings either for the $B$-violating terms, the $L$-violating terms, or both.

Thus, in the Minimal Supersymmetric Standal Model (MSSM), a discrete symmetry, $R$-parity [19], is customarily imposed which ensures the conservation of baryon as well as lepton number in the supersymmetric Lagrangian. Since there are strong indications that non-gauged symmetries are maximally violated by quantum gravity effects (leading to wormhole-induced proton decay [20|) whereas gauged symmetries are totally stable against such effects [21], we shall here assume that the proton-protecting symmetry is in fact a so-called discrete gauge symmetry [22]. In this case, it is of importance to note that although $R$ parity conservation leads to a comparatively simple phenomenology and a natural dark matter candidate (the LSP), the problem with proton decay is not satisfactorily solved when embedding the supersymmetric theory into more fundamental frameworks (such as GUTs) where baryon and lepton number violation can appear at some higher scale.

In an effective theory valid around the electroweak energy scale, the interactions mediated by super-heavy resonances associated with a higher-scale theory, take the form of non-renormalizable operators (i.e. operators of mass dimension $d \geq 5$ ) suppressed by $d-4$ powers of the high scale. Such operators, violating baryon and/or lepton number are generally not forbidden by $R$-parity conservation (see fig. 1), and it has been demonstrated that operators of dimension 5, being suppressed by only one power of the high (e.g. GUT) scale, will cause too rapid proton decay unless their couplings are suppressed by several orders of magnitude [23]. This should, by itself, provide a powerful argument for exploring alternatives to $R$-parity.

In [24], a systematic analysis of discrete gauge symmetries was carried out with the surprising conclusion 


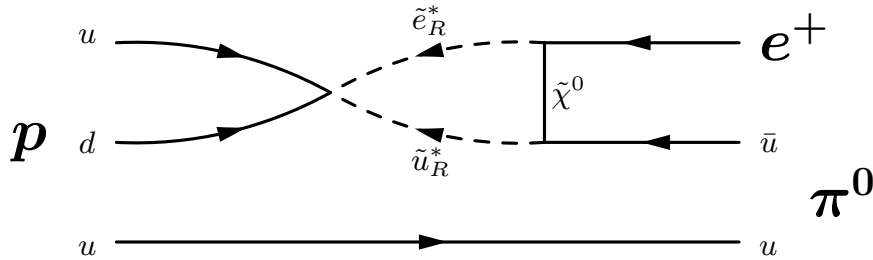

Fig. 1. An example of proton decay proceeding via a 5dimensional operator violating both $L$ and $B$ but conserving $R$. Due to the Majorana nature of the neutralino, it is drawn without an arrow.

that only $R$-parity and a $Z_{3}$ symmetry equivalent to $L$ conservation are anomaly-free, although some model dependence of this analysis 250 implies that also conservation of baryon number could be assured by viable symmetries. We shall here denote the two possibilities by $L$ parity and $B$-parity, though this should not be taken to mean that these symmetries stand for exact lepton number conservation and exact baryon number conservation (for obvious reasons to do with the matter-antimatter asymmetry of the universe). These names only reflect that in the supersymmetric part of the theory, the corresponding quantities are conserved. They may still be violated by GUT scale operators and will certainly be so by electroweak sphalerons. By far the most interesting observation, however, is that both $L$-parity and $B$-parity but not $R$-parity can forbid the dangerous dimension 5 contributions to proton decay.

Since a large number of current GUT and Planck scale theories do contain baryon and lepton number violation exterior to the supersymmetric MSSM framework, which will result in exactly this kind of contributions, it is important that experiments planning to explore SUSY are well prepared for the very distinct experimental signatures which are the hallmarks of $R$-parity violating scenarios, most importantly the consequences of a decaying LSP, of single sparticle production, and of the removal of the cosmological constraint that the LSP be neutral.

In this article, we assume the framework of the MSSM with the modification that lepton number is violated. This leads to the MSSM interaction Lagrangian being enlarged by the following terms [18]:

$$
\begin{aligned}
\mathcal{L}_{I}= & \lambda_{i j k}\left(\bar{\nu}_{L i}^{c} e_{L j} \tilde{e}_{R k}^{*}+\nu_{L i} \tilde{e}_{L j} \bar{e}_{R k}+\tilde{\nu}_{L i} e_{L j} \bar{e}_{R k}-i \leftrightarrow j\right) \\
& +\lambda_{i j k}^{\prime}\left(\bar{\nu}_{L i}^{c} d_{L j} \tilde{d}_{R k}^{*}+\nu_{L i} \tilde{d}_{L j} \bar{d}_{R k}+\tilde{\nu}_{L i} d_{L j} \bar{d}_{R k}\right. \\
& \left.\quad-\bar{e}_{R i}^{c} u_{L j} \tilde{d}_{R k}^{*}-e_{L i} \tilde{u}_{L j} \bar{d}_{R k}-\tilde{e}_{L i} u_{L j} \bar{d}_{R k}\right) \\
& \quad+\text { h.c. }
\end{aligned}
$$

where $i, j, k$ are generation indices (summation implied) and $\lambda_{i j k}$ is antisymmetric in its first two indices. The terms in the first line of the above equation are customarily denoted LLE terms and the terms in second LQD, in reference to the superfields appearing in the superpotential from which eq. (11) is derived. All of these terms contain two (SM) fermions and one (SUSY) scalar. The oddness under $R$ is thus directly visible here, since the SM fields have $R=+1$ and the SUSY fields $R=-1$. Also, it is clear that each sfermion can decay in a number of ways to two SM fermions via these couplings. $L$-violating neutralino, chargino, and gluino decays are forced to proceed via one or more intermediate scalar resonances.

A complete list of decay modes and full matrix elements for all decays of MSSM sparticles to SM particles can be found in [26]. Of these, we have implemented all but the gluino decays into the PYTHIA event generator, publically available from version 6.2 , with documentation included in the recently revised PyтнiA manual [27. The conclusions of the present paper are not expected to be changed significantly by the inclusion of $\Psi$ gluino decays since the gluino is typically heavy and thus has a number of other, unsuppressed decay channels available. Details about the PyтHia implementation can be found in [28].

Note that the sfermion decay matrix elements in $[26$ have been checked analytically by the author, and that the matrix elements listed there are not directly applicable in Pythia. Both Pythia and Herwig (IsAWIG) follow 29, 30 for the chargino and neutralino mixing conventions, but HERWIG uses the opposite convention [31] for the sfermion mixing angles, yielding a relative transposition between the two programs. In addition, extensive counter-checks were made between the two programs to make sure they agree円. Some non-negligible differences exist:

1. IsAWIG does not use running masses in the evaluation of higgsino-type couplings whereas PYTHIA does. This has been observed to lead to substantial differences between the widths calculated by the two programs (in rare cases factors of 2 or more).

2. $\alpha_{s}$ at $M_{Z}$ is used by IsAWIG in the calculation of the gluino decays. In the forthcoming update of PYTHIA where gluino decays are included, $\alpha_{s}$ will be evaluated at the mass of the gluino.

3. Pythia and Herwig (IsAsusy) do not use the same RGE's, so many parameters can differ quite substantially at the EW scale from the same GUT input, creating an "artificial" dissimilarity between the two programs. Ideally, one should compare models with identical EW scale mass spectra.

\section{3 mSUGRA Models}

The models used in this study have not been chosen among the ones initially suggested in the ATLAS Physics TDR [3]. This is partly due to the exclusion of most of these points by LEP (essentially from bounds on the Higgs mass), and partly since it is interesting to enable a direct comparison between the capabilities of the LHC and other, future experiments. The $5 \mathrm{mSUGRA}$ points shown in table 1 have therefore been selected among 14 points which were defined by the CLIC physics study group [32]. More recently - too late to be included in the studies reported here

1 This resulted in a few bug-fixes also in the HeRwIG code, so IsAwig has had a few bugs in the ME calculation up to and including v.1104. 
- a new set of standard benchmarks points were proposed [2] which have now been adopted by both the CLIC and other linear collider communities. See [2] for mass spectra analogous to table 1. To aid comparison, we briefly list the main differences/similiarities between our points and the new benchmark points.

- Point 'A' is a $(150 \mathrm{GeV})$ lighter version of $P_{2}$. The total SUSY (pair production) cross section is approximately 4 times larger than for $P_{2}: \sigma_{A} \approx 4 \sigma_{P_{2}}$.

- Points 'B', 'C', 'I', and particularly 'G' are similar to $P_{9} . \sigma_{P_{9}} \approx 0.5 \sigma_{B} \approx 5 \sigma_{C} \approx 2 \sigma_{I} \approx 3.5 \sigma_{G}$.

- Point ' $D$ ' is dissimilar to all our points. It is closest to $P_{2}$, but has a $500 \mathrm{GeV}$ lighter gluino, and $400 \mathrm{GeV}$ lighter squarks which increases the LHC cross section by an order of magnitude.

- Point ' $E$ ' is a lower-mass version of $F_{2}$, point ' $F$ ' a higher-mass version. $\sigma_{F_{2}} \approx 13 \sigma_{F} \approx 0.05 \sigma_{E}$.

- Point ' $\mathrm{H}$ ' is similar to $P_{7}$ but slightly heavier, giving a total LHC cross section $\sigma_{H} \approx \frac{1}{4} \sigma_{P_{7}}$.

- Point ' $\mathrm{J}$ ' is a high-tan $\beta$ version of $P_{2}$ with very close to identical total cross section. The mass hierarchies are also very similar.

- Point ' $\mathrm{K}$ ' is a large- $m_{1 / 2}$ sister of the only large-tan $\beta$ model included in this work, $P_{12}$. The correspondingly larger masses yield a cross section $\sigma_{K} \approx 0.04 \sigma_{P_{12}}$. The mass spectrum is similar to that of $P_{7}$.

- We have not included points at $\tan \beta=45$, but point 'M' can be seen as an even heavier version of point ' $\mathrm{K}$ ' $\approx P_{12}$ with a total cross section of only 0.1 femtobarns, and point ' $L$ ' has a mass spectrum not greatly different from $P_{9}$ but with heavier squarks and gluinos giving a cross section an order of magnitude lower than for $P_{9}$.

The total cross sections compared here include all MSSM pair production. Single sparticle production will give additional contributions depending on the strengths of the $R$-violating couplings.

One should keep in mind that all these points are defined for the MSSM, and as such have neutralino LSP's, a property which it has already been mentioned is not necessary in $\not R$-SUSY scenarios. Though we shall not do so here, it is certainly advisable to explore the experimental consequences of non-neutralino LSP's in more detail.

Both our points and the points in 2 assume a vanishing trilinear coupling at the GUT scale, i.e. $A_{0}=0$. In connection with this work, a small study of the direct consequences of that assumption upon the results presented here was performed. Varying $A_{0}$ between 0 and $500 \mathrm{GeV}$ for $P_{2}, P_{9}$, and $P_{12}$ gave only a weak variation $(\mathcal{O}(5 \%))$ of the semi-inclusive $L$-violating branching ratios (e.g. $B R\left(\tilde{\chi}_{1}^{0} \rightarrow q q \nu\right)$ ), and so apart from the consequences of the distortion of the mass spectrum caused by $A_{0} \neq 0$, the main signatures (number of leptons, number of jets, etc.) should be only mildly affected by changes to this parameter.

\footnotetext{
${ }^{2}$ Point 'H' gave a $\tilde{\tau}_{1}^{+}$LSP when using the GUT input parameters in [2] with ISASUGRA. We therefore chose a $30 \mathrm{GeV}$ larger value for $m_{0}$, retrieving $\tilde{\chi}_{1}^{0}$ as the LSP.
}

\begin{tabular}{|c|c|c|c|c|c|}
\hline & $\mathbf{P}_{2}$ & $\mathbf{P}_{7}$ & $\mathbf{P}_{9}$ & $\mathbf{P}_{12}$ & $\mathbf{F}_{2}$ \\
\hline \multicolumn{6}{|c|}{ GUT Parameters } \\
\hline $\tan \beta$ & 5 & 10 & 20 & 35 & 10 \\
\hline$m_{0}$ & 170 & 335 & 100 & 1000 & 2100 \\
\hline$m_{\frac{1}{2}}$ & 780 & 1300 & 300 & 700 & 600 \\
\hline $\operatorname{sign}^{2}(\mu)$ & + & + & + & - & + \\
\hline$A_{0}$ & 0 & 0 & 0 & 0 & 0 \\
\hline \multicolumn{6}{|c|}{ Mass Spectrum } \\
\hline$h^{0}$ & 118 & 123 & 115 & 120 & 119 \\
\hline$A^{0}, H^{ \pm}, H^{0}$ & 1100 & 1663 & 416 & 944 & 2125 \\
\hline$\tilde{\chi}_{1}^{0}$ & 325 & 554 & 118 & 293 & 239 \\
\hline$\tilde{\chi}_{2}^{0}, \tilde{\chi}_{1}^{+}$ & 604 & 1025 & 217 & 543 & 331 \\
\hline$\tilde{\chi}_{3}^{0}$ & 947 & 1416 & 399 & 754 & 348 \\
\hline$\tilde{\chi}_{4}^{0}, \tilde{\chi}_{2}^{+}$ & 960 & 1425 & 416 & 767 & 502 \\
\hline$\tilde{g}$ & 1706 & 2752 & 707 & 1592 & 1442 \\
\hline$\tilde{e}_{R}, \tilde{\mu}_{R}$ & 336 & 584 & 156 & 1031 & 2108 \\
\hline$\tilde{\tau}_{1}$ & 334 & 574 & 126 & 916 & 2090 \\
\hline$\tilde{e}_{L}, \tilde{\mu}_{L}$ & 546 & 917 & 231 & 1098 & 2126 \\
\hline$\tilde{\tau}_{2}$ & 546 & 915 & 240 & 1051 & 2118 \\
\hline$\tilde{\nu}$ & 541 & 913 & 217 & 1095 & 2125 \\
\hline$\tilde{q}_{R}$ & 1453 & 2333 & 612 & 1612 & 2328 \\
\hline$\tilde{b}_{1}$ & 1403 & 2262 & 566 & 1412 & 2010 \\
\hline$\tilde{t}_{1}$ & 1189 & 1948 & 471 & 1241 & 1592 \\
\hline$\tilde{q}_{L}$ & 1514 & 2425 & 633 & 1663 & 2343 \\
\hline$\tilde{b}_{2}$ & 1445 & 2312 & 615 & 1482 & 2310 \\
\hline$\tilde{t}_{2}$ & 1443 & 2286 & 648 & 1451 & 2018 \\
\hline \multicolumn{6}{|c|}{ LHC Parameters } \\
\hline & $\mathbf{P}_{2}$ & $\mathbf{P}_{7}$ & $\mathbf{P}_{9}$ & $\mathbf{P}_{12}$ & $\mathbf{F}_{2}$ \\
\hline$\sigma_{\mathrm{SUSY}}[\mathrm{fb}]$ & 130 & 3.9 & 24000 & 110 & 110 \\
\hline$\times 30 \mathrm{fb}^{-1}$ & 3900 & 114 & 720000 & 3300 & 3300 \\
\hline
\end{tabular}

Table 1. Selected points of analysis in the mSUGRA parameter space, mass spectra as obtained with IsASUSY, and total SUSY pair production cross section at the LHC.

In addition to the mSUGRA parameters come the $9 \lambda$ (LLE) and the $27 \lambda^{\prime}$ (LQD) couplings for which the scenarios listed in table 2 have been studied for each mSUGRA point. The reason we do not consider couplings larger than $10^{-2}$ is partly due to the present experimental bounds [33] and partly due to the fact that some implicit approximations in this analysis would break down for larger couplings: 1) the $\not R$ couplings are not included in the RGE evolution of the SUSY parameters and 2) single sparticle production is not simulated. Note that the large-coupling (a) scenarios only observe the limits on individual couplings given in [33], not the limits on products of the couplings. Thus, these scenarios are useful for studying the consequences of having many large couplings, but one should keep in mind that they are unrealistic in that not all of the couplings can be simultaneously large.

On the other hand, if the $\not R$ couplings get significantly smaller than $10^{-4}$, the LSP lifetime can become so large that it decays outside the detector, mimicking the $R$ conserving scenarios which have already been extensively studied. For example, for the mSUGRA point $F_{2}$, setting 


\begin{tabular}{cccc}
\hline & \multicolumn{4}{c}{$\mathbf{C}$ O U P L I N G } & M O D E L S \\
& $a$ & $b$ & \\
\hline \multicolumn{4}{c}{ L L E } \\
$\lambda_{i j k}$ & $10^{-2}$ & $10^{-4}$ & $\sqrt{\hat{m}_{e_{i}} \hat{m}_{e_{j}} \hat{m}_{e_{k}}}$ \\
$\lambda_{i j k}^{\prime}$ & 0 & 0 & 0 \\
\hline \multicolumn{5}{c}{$\mathrm{L} \mathrm{Q} \mathrm{D}$} \\
$\lambda_{i j k}$ & 0 & 0 & 0 \\
$\lambda_{i j k}^{\prime}$ & $10^{-2}$ & $10^{-4}$ & $\sqrt{\hat{m}_{e_{i}} \hat{m}_{q_{j}} \hat{m}_{d_{k}}}$ \\
\hline \multicolumn{5}{c}{$\mathrm{L} \mathrm{L} \mathrm{E}+\mathrm{L} \mathrm{Q} \mathrm{D}$} \\
$\lambda_{i j k}$ & $10^{-2}$ & $10^{-4}$ & $\sqrt{\hat{m}_{e_{i}} \hat{m}_{e_{j}} \hat{m}_{e_{k}}}$ \\
$\lambda_{i j k}^{\prime}$ & $10^{-2}$ & $10^{-4}$ & $\sqrt{\hat{m}_{e_{i}} \hat{m}_{q_{j}} \hat{m}_{d_{k}}}$ \\
\hline
\end{tabular}

Table 2. Selected points of analysis in the $\lambda-\lambda^{\prime}$ parameter space. The last column corresponds to the "natural coupling" scenario proposed in 23]. $\hat{m} \equiv \frac{m}{v}=\frac{m}{126 \mathrm{GeV}}$ and $m_{q_{j}} \equiv \frac{1}{2}\left(m_{u_{j}}+m_{d_{j}}\right)$. These models (coloumn $n$ ) will be referred to as "nLLE", "nLQD", and "nLLE + nLQD" in the text below.

all $\lambda$ couplings to $10^{-6}$ and all $\lambda^{\prime}$ couplings to zero results in a decay length for the LSP of $\tau c=40 \mathrm{~m}$. In the intermediate range, one may see the LSP decay directly inside the fiducial volume of the detector (see e.g. [34]), yet we abstain from relying on such a spectacular signature here so as not to be overly optimistic in our results.

\section{Triggers for $\not$-SUSY}

A reasonable aim for the total $\amalg$-SUSY dedicated trigger rate is about $1 \mathrm{~Hz}$. We here focus on rates after the events have been filtered through the trigger system, i.e. we make no distinction between trigger levels. This is a technical issue which requires more detailed knowledge of the detector performance at mid-luminosity than is currently parametrized in ATLFAST. Specifically, no parametrization of the effects of pile-up at mid-luminosity is included, and so we here adopt a best-guess approach, performing the simulation without pile-up, and then multiplying the resulting trigger rates by a factor of $5 / 3$ to estimate the true rates at $\mathcal{L}=3 \times 10^{33} \mathrm{~cm}^{-2} \mathrm{~s}^{-1}$. This factor is based on the scaling exhibited by the inclusive electron, electron/photon, and $\not_{T}+2$ jets trigger rates presented in [3, chp.11] from low to high luminosity.

To retain as much generality as possible in the trigger definitions, it is sensible to use the information contained in the $\angle$ superpotential terms rather than a selection of decay modes to define the trigger menus. The $\lambda$ (LLE) couplings are purely leptonic, and thus single sparticle production via these terms is not relevant for the LHC. Thus we are searching for at least two hard leptons (which, however, may be tauons), in most cases accompanied by $\oiint_{T}$ from escaping neutrinos. For the $\lambda^{\prime}$ (LQD) terms, we expect at least two leptonic objects accompanied by hard jets when the dominant production mechanism is MSSM pair production. Note that pure jet signatures would also be possible through single slepton production, $q_{1} \bar{q}_{2} \rightarrow \tilde{\ell} \rightarrow q_{3} \bar{q}_{4}$. Single squark production

\begin{tabular}{lrrr}
\hline \multicolumn{4}{c}{ EVENT RATES AND SAMPLE SIZES } \\
\hline Process & $\boldsymbol{\sigma}[\mathrm{mb}]$ & Rate $[\mathbf{H z}]$ & $\boldsymbol{N}_{\boldsymbol{g e n}}$ \\
QCD $2 \rightarrow 2$ & & & \\
$p_{T}=1-10 \mathrm{GeV}$ & 55 & $1.6 \times 10^{8}$ & $2.5 \times 10^{8}$ \\
$p_{T}=10-75 \mathrm{GeV}$ & 12 & $3.7 \times 10^{7}$ & $2.2 \times 10^{8}$ \\
$p_{T}=75-150 \mathrm{GeV}$ & $5.5 \times 10^{-3}$ & $1.7 \times 10^{4}$ & $1.4 \times 10^{7}$ \\
$p_{T}>150 \mathrm{GeV}$ & $2.9 \times 10^{-4}$ & $8.7 \times 10^{2}$ & $1.1 \times 10^{7}$ \\
\hline$t \bar{t}$ & $6.2 \times 10^{-7}$ & 1.9 & $5.9 \times 10^{6}$ \\
$Z / W$ & $1.2 \times 10^{-3}$ & $3.6 \times 10^{3}$ & $1.8 \times 10^{8}$ \\
$Z Z / Z W / W W$ & $1.2 \times 10^{-7}$ & 0.36 & $5.9 \times 10^{6}$ \\
\hline
\end{tabular}

Table 4. Numbers of generated events for the trigger study. The rates listed are total rates before trigger for $\mathcal{L}=3 \times$ $10^{33} \mathrm{~cm}^{-2} \mathrm{~s}^{-1}$. QCD events with the $p_{T}$ of the hard interaction below $1 \mathrm{GeV}$ were not simulated.

via $L Q D$ requries a lepton in the initial state and is thus suppressed at the LHC.

The triggers so far investigated are listed in table 3 , where background rates and efficiency ranges for all models investigated are shown. For comparison, results for MSSM scenarios are also given. The event generation was performed with an augmented version of PyтHIA 6.155 [35] and AtLFast v.2.53 [36]. With respect to AtLFAst, an attempt was made at obtaining more believable muon and electron reconstruction efficiencies by including by hand a muon efficiency of $95 \%$ independent of muon momentum and an electron reconstruction efficiency of $80 \%$ for electrons with $p_{T}>50 \mathrm{GeV}$ and $70 \%$ for electrons with $p_{T}<50 \mathrm{GeV}$. These estimates are based on the ATLAS Physics TDR [3].

Background cross sections, pre-trigger event rates, and sample sizes are listed in table 4 . Though low- $p_{T}$ QCD events have essentially no chance to pass triggers and much less the subsequent analysis cuts, the very high purities required for SUSY signal extraction do not immediately admit these events to be discounted entirely. Rather, a substantial sample of such events was generated with the object of placing an upper bound on the number of low- $p_{T}$ QCD events remaining after cuts using Poisson statistics. This will be discussed in section f 5 , however some cautiousness should be employed in interpreting the bounds obtained, since the Monte Carlo is here being stretched far into the tails of its $p_{T}$ distributions.

We do not consider triple gauge boson production. The cross section (excluding Higgs-induced production) is around $100 \mathrm{fb}$ [37, i.e. at the same level as interesting SUSY cross sections, yet invariant mass cuts on pairs of jets would presumably be able to reduce this background considerably, and so we do not believe that these processes are dangerous as background sources.

More detailed remarks and a large selection of plots of trigger rates and efficiencies versus thresholds can be found in 28].

From the efficiencies in table 3, one easily sees how much cleaner the signatures of the purely leptonic (LLE) coupling are compared to the signatures involving quarks (LQD) where higher thresholds, due to the hadronic envi- 


\begin{tabular}{lcrrr}
\hline Trigger & $\begin{array}{c}\text { Background } \\
\text { Rate }\end{array}$ & $\begin{array}{c}\text { MSSM } \\
\text { Efficiency }\end{array}$ & $\begin{array}{c}\text { LLE } \\
\text { Efficiency }\end{array}$ & $\begin{array}{c}\text { LQD } \\
\text { Efficiency }\end{array}$ \\
\hline mu45I + mu45I & $0.2 \mathrm{~Hz}$ & $1-5 \%$ & $10-40 \%$ & $1-10 \%$ \\
e45I + e45I & $0.1 \mathrm{~Hz}$ & $1-5 \%$ & $1-35 \%$ & $1-10 \%$ \\
mu15I + e15I & $0.1 \mathrm{~Hz}$ & $2-5 \%$ & $20-60 \%$ & $2-15 \%$ \\
mu40I + me75 & $0.3 \mathrm{~Hz}$ & $10-25 \%$ & $40-75 \%$ & $10-35 \%$ \\
e40I + me75 & $0.2 \mathrm{~Hz}$ & $10-20 \%$ & $15-70 \%$ & $10-35 \%$ \\
j100 + mu40I & $0.5 \mathrm{~Hz}$ & $10-20 \%$ & $45-70 \%$ & $10-40 \%$ \\
j100 + e40I & $0.5 \mathrm{~Hz}$ & $5-15 \%$ & $15-65 \%$ & $10-35 \%$ \\
j100 + me175 & $0.3 \mathrm{~Hz}$ & $50-80 \%$ & $35-80 \%$ & $25-80 \%$ \\
$3 \mathrm{j} 50+$ mu20I & $0.1 \mathrm{~Hz}$ & $5-15 \%$ & $45-60 \%$ & $12-40 \%$ \\
$3 \mathrm{j} 50+$ e30I & $0.1 \mathrm{~Hz}$ & $5-10 \%$ & $15-55 \%$ & $10-35 \%$ \\
$3 \mathrm{j} 75+$ me125 & $0.1 \mathrm{~Hz}$ & $30-65 \%$ & $30-70 \%$ & $40-90 \%$ \\
\hline Total Rate & $2.1 \mathrm{~Hz}$ & $60-90 \%$ & $90-99.9 \%$ & $60-96 \%$ \\
/Combined Efficiency & & & & \\
\hline
\end{tabular}

Table 3. Estimated trigger rates for background processes and trigger efficiency ranges for the various MSSM points and $\not /-$ SUSY scenarios studied for $\mathcal{L}=3 \times 10^{33} \mathrm{~cm}^{-2} \mathrm{~s}^{-1}$. The nomenclature follows the ATLAS standard, where e.g. "mu45I" means an isolated muon with $p_{T}>45 \mathrm{GeV}$, "me" stands for missing (transverse) energy, and " $3 \mathrm{j} 50$ " means 3 jets, each with $p_{T}>50 \mathrm{GeV}$. The total rate is smaller than the sum of the individual rates since there is a certain overlap, and the combined efficiencies can be larger than the individual efficiencies, since there is not a total overlap between the triggers.

ronment, mean smaller efficiencies. From this we conclude that it would be of interest to extend the LQD study, examining whether 2-jet triggers and/or 3-object triggers could enhance the efficiency.

Lastly, though the trigger proposals given here are designed explicitly with $\not$-SUSY in mind, they show a certain overlap with triggers proposed for more conventional physics. The di-muon and 3 jets + lepton triggers, for example, have also been proposed for various Higgs searches. The di-electron trigger as well as 3 jets + electron are proposed to catch $t \bar{t}$ decays. Finally, the conventional SUSY searches also make use of multi-lepton, jets $+\not_{T}$, and multi-jet signatures 38]. It is therefore not unlikely that the triggers proposed here can be incorporated to some extent into the conventional trigger programme.

\section{Discovery of $\not L$-SUSY at the LHC}

The main purpose here is to deliver an impression of what kind of signal strengths can be achieved at the LHC with $30 \mathrm{fb}^{-1}$ integrated luminosity. To maintain generality in view of the more than thousand $L$-violating channels possible, we do not discuss invariant mass reconstruction or measurements of the SUSY parameters in general. We focus entirely on the isolation of candidate events through inclusive and kinematic event-shape variables in the attempt to obtain a statistically significant signal as compared with the background expectation after cuts. We do this for several models simultaneously (using neural networks in the final step), an approach which is complementary to the conventional one, where specific scenarios are studied one by one.

Note that no attempt is made, beyond a crude worstcase estimate, to include the effects of pile-up in this analysis.

\subsection{Missing Transverse Energy}

The post-trigger $\not_{T}$ distribution for the SM and its composition is shown in figure 2a. Note that there are so few double gauge boson events that they are hardly visible on the plot. The peaks at $\not_{T}=75 \mathrm{GeV}$ and at $\not_{T}=175$ are due to the me75 and me175 triggers becoming active.

In figure $2 \mathrm{~b}$ the distributions for the most "low-mass" mSUGRA point, $P_{9}$, are shown for $P_{9}$ (MSSM), $P_{9 a}$ (LLE), and $P_{9 a}(\mathrm{LQD})$. The degradation of the $E_{T}$ signature in the If models is evident, though one observes by comparing with fig. 2a that a cut at low $\not_{T}$ values is still possible. Note that the $\not_{T}$ trigger peaks mentioned above are absent for the LLE scenario, this simply because the LLE scenarios do not rely to so great an extent on the $\mathbb{E}_{T}$ triggers, c.f. table 3 .

The full range of mSUGRA models are plotted in figure 2c. To enable the models to be shown on the same scale and to be distinguished from each other, the histograms have been normalized to unit area and smoothed. These plots are not intended to give detailed information, but rather to illustrate the spread between the models. Models with heavy squarks and gluinos $\left(P_{7}, F_{2}\right.$, and to some extent $P_{12}$ ) have rather flat, MSSM-like signatures whereas the models with lighter sparticles are peaked at low $\not_{T}$. This is an important point, since the light-sparticle scenarios have high production cross sections and the heavysparticle ones low cross sections. A cut on $\not_{T}>100 \mathrm{GeV}$ seems a reasonable compromise between losing events in the peaked distributions (where we have many events anyway) and efficient background rejection required for the heavier scenarios (where we lose little by the cut but have fewer events). 

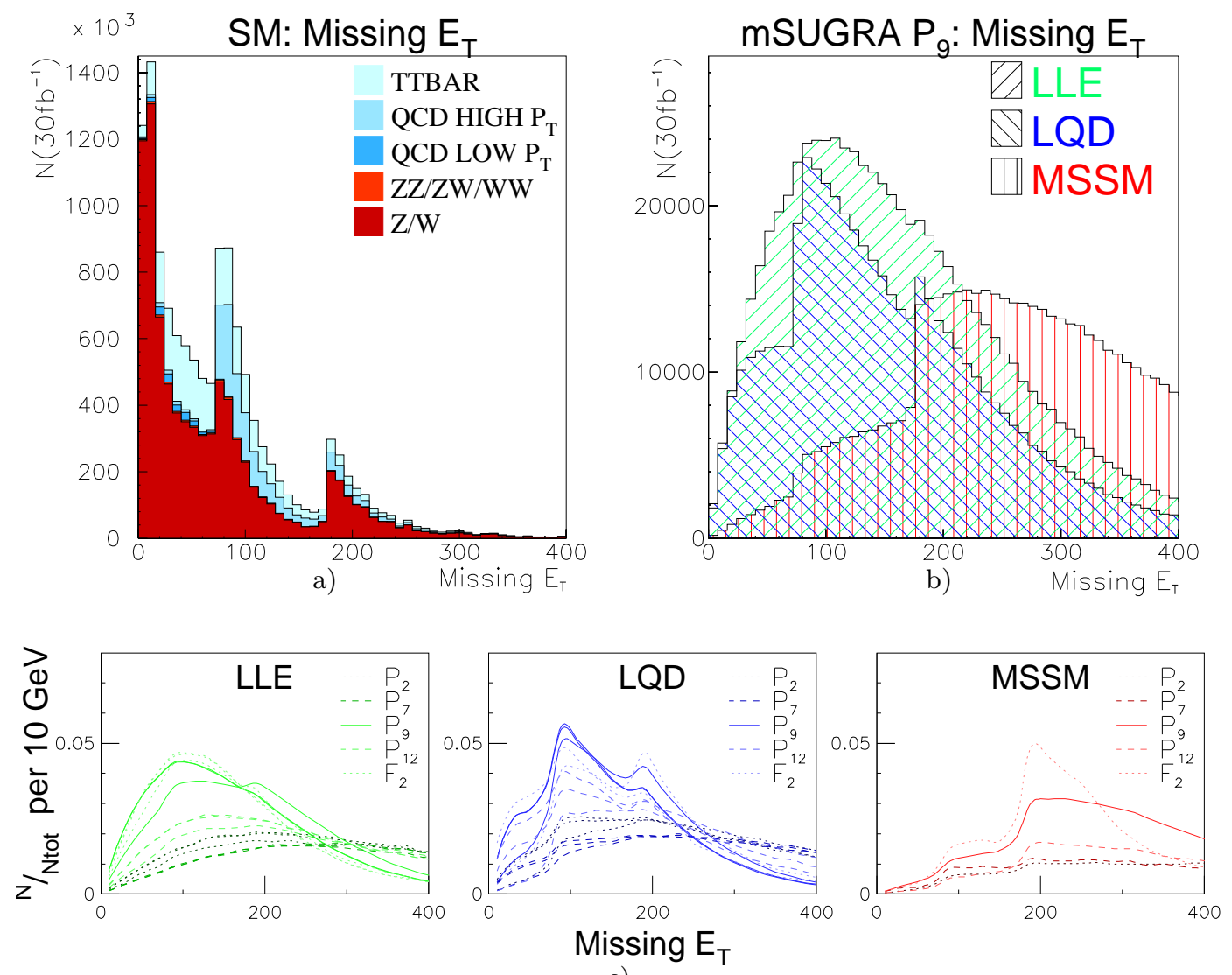

c)

Fig. 2. a) and b): $\not \not_{T}$ signatures for the SM and mSUGRA point $9 a$ models (i.e. all relevant $\not R$ couplings set to $10^{-2}$ ) normalized to $30 \mathrm{fb}^{-1}$ of data taking. "QCD LOW $p_{T}$ " means events from the $100 \mathrm{GeV}<p_{T}<150 \mathrm{GeV}$ sample and "QCD HIGH $p_{T}$ " events from the $p_{T}>150 \mathrm{GeV}$ sample. c): Event distributions normalized to unit area for LLE, LQD, and the MSSM for all mSUGRA and $\not /$ coupling points studied. The last row of plots is not intended to give detailed information, merely to illustrate the spread between the models.

\subsection{Leptons and Jets}

Due to the fact that the LSP decays, one expects an increased number of leptons and/or jets in the $I$-SUSY scenarios. In figures 3 and 1 the number of leptons is plotted versus the number of jets for each of the SM backgrounds and for $P_{9}(\mathrm{MSSM}), P_{9 a}(\mathrm{LLE})$, and $P_{9 a}(\mathrm{LQD})$. All events satisfy $\not_{T}>100 \mathrm{GeV}$.

The absolute normalizations are, of course, very different for each of the SM plots.

One notices that the number of reconstructed jets goes up to 15 on the plots in figs. 3 and 4 . The jet numbers are obtained using the ATLFAST standard cone algorithm jet finder, employing a cone size of $\Delta R=\sqrt{(\Delta \phi)^{2}+(\Delta \eta)^{2}}=$ 0.4 and with clusters up to pseudorapidities $|\eta|<5$ included only if their transverse energies are above $E_{T}^{\text {min }}=$ $10 \mathrm{GeV}$. Whether a resolution of up to 15 jets or more is possible (and reliable) in the finished experiment is questionable, yet the important point remains that there is more hadronic activity associated with the LQD scenarios than is the case for the MSSM, and we should be able to distinguish between the two possibilities using any jet finder.
The increased number of leptons in the LLE scenario as well as the increased number of jets (and a small increase in the number of leptons) in the LQD scenario relative to the MSSM are evident. Since the $Z / W$ and high- $p_{T}$ QCD backgrounds have the highest cross sections, a cut removing events in the lower left corner was performed, requiring $N_{\text {Jets }}+N_{\ell} \geq 8$ or $N_{\ell} \geq 3$. This cut has a rejection factor of about 20 for the SM events and efficiencies above $75 \%$ for all IL-SUSY scenarios, again with a tradeoff between signal loss at low-mass points and efficient background rejection for high-mass points.

\subsection{Additional Variables}

In addition to the above mentioned requirements, cuts on several other kinematical variables were performed before the neural network analysis below. We shall here only briefly define and comment these variables (see [28] for details):

- The $p_{T}$ of the hardest object (jet or lepton) in the event was required to be greater than $200 \mathrm{GeV}$. 

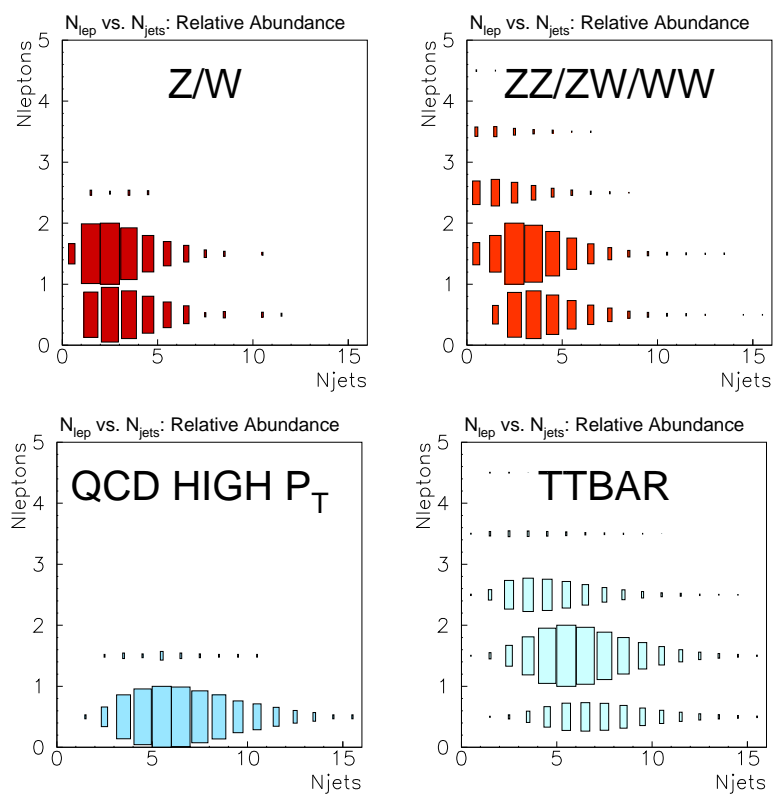

Fig. 3. Lepton versus jet multiplicity (see text) for background events surviving the cut on $\not_{T}$. The numbering of the bins is such that the events with 0 jets are in the bin to the right of the number 0 and events with 0 leptons are in the bin above the number 0 .
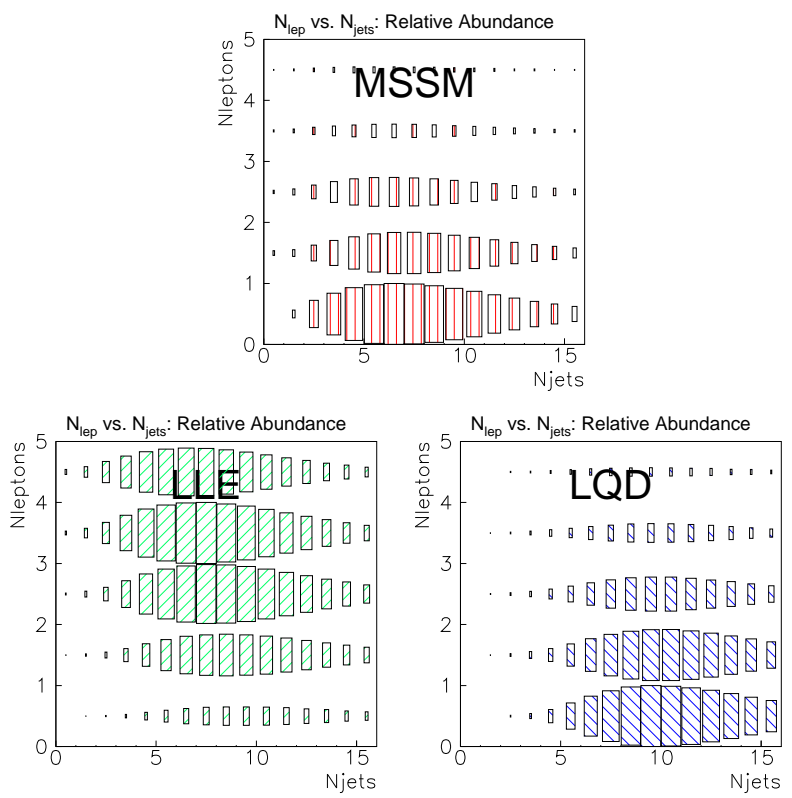

Fig. 4. Lepton versus jet multiplicity (see text) in mSUGRA $P_{9}$ for events surviving the cut on $\not_{T}$. The numbering of the bins is such that the events with 0 jets are in the bin to the right of the number 0 and events with 0 leptons are in the bin above the number 0 .
- We define the " $p_{T}$-weighted 4-object energy correlation" by:

$$
P_{4 C} \equiv \frac{1}{12}\left(\frac{E_{4}}{E_{3}}+\frac{E_{3}}{E_{2}}+\frac{E_{2}}{E_{1}}\right)\left(p_{T 1}+p_{T 2}+p_{T 3}+p_{T 4}\right)
$$

where $E_{1}, \ldots, E_{4}\left(p_{T 1}, \ldots, p_{T 4}\right)$ are the energies (transverse momenta) of the four hardest objects in the event. For pair-produced LSP's, one would expect four hard objects with more or less equal energies more often than would happen for background events. The energy fractions are weighted by the average $p_{T}$ to give the variable some extra sensitivity against $t \bar{t}$ events. A cut at $P_{4 C}>200 \mathrm{GeV}$ was placed.

- Events with Thrust greater than 0.85 were rejected. The Thrust calculation naturally suffers from the loss of particles down the beam-pipe at high pseudorapidities. Only particles with $|\eta|<5$ are defined as being inside the active calorimetry range in ATLFAST, and only these particles are included in the Thrust calculation.

- Events with Oblateness greater than 0.4 were rejected P. $^{2}$.

- Events with Circularity less than 0.1 were rejected In $^{2}$.

\subsection{Neural Network Cuts}

It is clear that the above cuts do not define a dedicated search strategy. They were applied to all scenarios with no distinction between MSSM, LLE, and LQD models. A more comprehensive analysis would of course have to focus on each of these possibilities separately, and also some differentiation between the mSUGRA parameters would be required. In particular sparticles lighter than the top and ones heavier than the top could with advantage be searched for using separate strategies.

This highlights the fact that the mSUGRA parameter space, including now the $I /$ couplings, is not small. It was judged an uneconomical use of resources at this point to optimize a purely physics-based analysis for each of the many possibilities offered by this large parameter space. Instead, three neural networks were trained with post-trigger events to separate MSSM, LLE, and LQD scenarios, respectively, from the background distributions. Each network was thus trained not with one scenario but with equal numbers of events from 15 different scenarios with varying mSUGRA parameters and varying $\not L-$ coupling strengths, allowing the networks to pick out general qualities common to each class without over-fitting to a particular model.

This procedure is certainly prone to the dangers which are always present when using neural networks on simulated data, in that the input parameters (simulated experimental observables) do not come with warning labels about where in their domains the simulated results can

\footnotetext{
3 Note that Oblateness is calculated entirely in the transverse plane for hadron colliders.

4 Same comment as for Oblateness.
} 
be trusted and where not. However, exercizing caution on this point, the method proposed here could be a useful alternative for exploring theories with large parameter spaces.

As inputs were used all of the above mentioned variables along with the $p_{T}$ of the four hardest jets and the two hardest leptons. The networks employed were single-layer perceptrons using the gradient descent learning algorithm, with biased linear input and output neurons, and hidden neurons activated according to a biased sigmoid ('logistic') function. At the end of each learning period, the networks were trimmed using the OBD (Optimal Brain Damage) prescription [39]. After ten such periods, the networks generally showed a negligible difference in performance on the learning sample as compared with the performance on an independent monitor sample, indicating that over-fitting to the training samples is not a problem.

The training samples consisted of 3000 post-trigger SUSY events, selected equally among the appropriate scenarios, and 4000 background events, weighted according to their post-trigger cross sections to represent approximately $10^{7}$ events in the learning algorithm. An output of zero (one) was the target for background (SUSY) events.

The finished LLE (LQD) network showed a clear preference for low-Thrust, multi-lepton (multi-jet), low-Oblateness events with a mixture of low and high $\not_{T}$. For slightly higher Thrust values, low lepton momenta were preferred in the LLE case. For the LQD network, the most noteworthy additional feature was a strong relaxation of all other considerations for very high jet energies. Note that the background levels at low Thrust values suffer from some theoretical uncertainty due to the fact that parton showers have been used to generate the multi-jet final states, an approach which in the past has been known to give too few multi-jet events. Though it is not clear that present generators suffer from the same problems, it is important to note that there is a non-negligible and potentially dangerous uncertainty in any analysis relying on parton-shower simulations for large jet numbers and low Thrust values.

Processing now the full event samples remaining after the cuts described above through each of the three (MSSM, LLE, and LQD) networks, a final cut was made requring outputs larger than 0.9 . Since the learning samples were small compared to the full event samples (e.g. about 100.000 SUSY events were generated per scenario and only a few hundred used for training) no effort was made to exclude those events which had participated in the learning process from the analysis. This is, in principle, a source of error, yet we permit ourselves to ignore it due to the smallness of the learning samples and since any problems related to over-fitting have been minimized by OBD. For the SM backgrounds, the generated event samples had been depleted considerably by the initial cuts, and only the $t \bar{t}$ and double gauge boson samples had any events remaining at all after the network cuts (note that the learning samples used for training - for both background and SUSY - were post-trigger events).

The procedure used to construct upper bounds on the actual event numbers was, for $N$ events remaining in a sample, to calculate the mean, $\mu$, of the Poisson distribution which has exactly $5 \%$ chance to result in $N$ or fewer events. $\mu$ is then interpreted as a "95\% CL" upper bound on the number of events which could have passed the cut. For the low- $p_{T}$ QCD events, this number was then subjected to the same rejection factors under cuts as the high- $p_{T}$ sample (later the rejections for the $t \bar{t}$ sample were used for both), and the double gauge boson rejection factors were used for the upper bound on single gauge events.

Typically, for $30 \mathrm{fb}^{-1}$, between 500 and 1000 signal events remain after cuts for $P_{2}, P_{12}$, and $F_{2}$. Many more of course remain for $P_{9}$ because of the larger cross section, but there is no hope for $P_{7}$ with only 114 events expected in total in $30 \mathrm{fb}^{-1}$ of data. Yet one should bear in mind that single sparticle production which has not been included here could significantly increase the cross section if the $\not R$ couplings are not much smaller than the gauge couplings. For a hadron machine like the LHC, this effect would only be big for the LQD terms since single slepton resonances would then be possible. Of course, if $\not B$ terms are present, single squark production would be possible as well.

\subsection{Results}

We define the statistical significance with which a discovery can be made by

$$
P=\frac{S}{\sqrt{S+B}}
$$

and conservatively interpret $P>5$ rather than the conventional $S / \sqrt{B}>5$ to mean that a $5 \sigma$ discovery will be possible. Using the event numbers obtained in the analysis (the estimates on $B$ being $95 \%$ CL upper bounds) results in the $P$ values listed in table 5 .

In reality, $P$ depends on (unknown) systematic QCD uncertainties (parton distributions etc) and should be corrected for the effects of pile-up, and so we can only be confident that a $5 \sigma$ discovery is possible if $P$ is somewhat larger than 5 . Therefore, aside from working with the definition, eq. (3), we attempt to define a more pessimistic quantity in a very crude, ad hoc manner which we shall call $P_{\text {corr }}$.

The non-inclusion of pile-up results in too optimistic estimates of $S / B$. To include an estimate of the reduction of this ratio, we rewrite eq. (3) to:

$$
P=\frac{\sqrt{S}}{\sqrt{1+B / S}}
$$

where we now include the effects of pile-up by multiplying $B / S$ by some factor. That twice as many background events per signal event could be passing the analysis if pile-up was included seems a reasonably pessimistic guess.

Furthermore, assuming that the intrinsic uncertainty on both $S$ and $B$ coming from uncertainties on QCD parameters will, to a first approximation, work in the same direction and with a comparable magnitude for both $B$ 
and $S$, we expect that the denominator in the above formula is not affected by this uncertainty, and so we include the QCD-related uncertainties by reducing the number of signal events in the numerator by a factor of 1.5 , believing this to be an adequate worst-case estimate. This yields the following form for the "corrected discovery potential":

$$
P_{\text {corr }}=\frac{S}{\sqrt{1.5 S+3 B}}
$$

Of course, this quantity should not be taken too seriously. We list it in table 5 merely to show the effects of the stated factors on the discovery potential, i.e. a reduction of $S / B$ by a factor of 2 combined with a reduction of both $S$ and $B$ by $2 / 3$.

With regard to the sensitivity of this analysis on systematic uncertainties on the normalization of $B$, note that only for $P_{9}$ do we have an $S / B \gg 1$. For the $P_{2}, P_{12}$, and $F_{2}$ scenarios $S / B$ lies between one half and unity. We do not estimate this to be a serious problem since our background estimate is only a 95\% CL upper limit (larger event samples would most likely bring the high- $p_{T}$ QCD component down) and since the background normalization can presumably be determined to better than a factor 2 (as is used in $P_{\text {corr }}$ ) using complementary regions of phase space where the SUSY population is low or vanishing.

What one can see in table 5 is again that the LLE scenarios typically yield much purer event samples than the LQD ones, due to the hadronic environment at the LHC. It is also worth noticing that it is not easy, from this analysis alone, to discriminate between MSSM, LLE, and LQD scenarios. This is due to the fact that the three networks were trained independently, i.e. they were taught to reject SM events but not events from the other scenario types.

A suggestion for how we might have gained further insight would be to construct one network with one output node for each class of models, requiring an output of $(0,0, \ldots)$ for background events, $(1,0,0, \ldots)$ for events of class $1,(0,1,0,0, \ldots)$ for events of class 2 , and so on. One would then be able to construct a measure for the relative probability of the events surviving cuts belonging to a given class of models, by comparing their $n$-dimensional distribution in the network output space with the expected background distribution.

\section{Outlook and Conclusion}

\subsection{Outlook:}

Though some preliminary studies have been performed in the present work, the field is large and many important tasks remain. Recently, a significant theoretical effort has been dedicated to studying the case of baryon number violation 40,41,42. Both single sparticle production and baryon number violating SUSY will be included in the PYTHIA generator in the foreseeable future.

This will hopefully spur more LHC activity, with comprehensive studies of both lepton and baryon number violating scenarios. Preliminary studies indicate a lessening of the reach of the LHC in baryon number violating scenarios 40], and it would be of interest to explore this reach with the full range of production and decay mechanisms included. In addition, studies should certainly be carried out for mSUGRA benchmark points without neutralino LSP's.

Finally, triggers dedicated for $\not R$-SUSY should be incorporated in the standard ATLAS trigger programme.

\subsection{Conclusion:}

1278 decay modes of Supersymmetric particles into Standard Model particles through lepton number violating couplings have been implemented in the PYTHIA event generator. Combining this augmented version of the generator with a crude simulation of the ATLAS detector, trigger menus for mid-luminosity running of the LHC have been proposed and seen to have a high acceptance of supersymmetric events in several $L$-violating SuperGravity scenarios while still giving event rates in the $1 \mathrm{~Hz}$ region.

Taking these trigger menus as basis, the possibility for a $5 \sigma$ discovery after $30 \mathrm{fb}^{-1}$ data taking was estimated for each investigated model, using a technique based on simultaneous classification of groups of models rather than detailed studies of single models.

For cross sections down to $10^{-10} \mathrm{mb}$ (or, roughly, for points with $m_{0}<2 \mathrm{TeV}$ and $m_{1 / 2}<1 \mathrm{TeV}$ ) it was found that a $5 \sigma$ discovery was possible for all scenarios with $30 \mathrm{fb}^{-1}$ of data. It is estimated that uncertainties related to QCD parameters or pile-up in the detector, both of which have not been taken into account in the present analysis, could not significantly affect this conclusion.

$L$-violating decays of the gluino and the possibility of invoking $B$-violation will be implemented in the PYTHIA generator within a foreseeable future.

Acknowledgement: The author gratefully acknowledges support from Frederikke Lørup f. Helms Mindelegat and thanks P. Richardson and S. Mrenna for helpful correspondence.

\section{References}

1. R. Haag, J. T. Lopuszanski, M. Sohnius, Nucl. Phys. B 88, 257 (1975)

2. M. Battaglia et al. [hep-ph/0106204]

3. ATLAS Collaboration, CERN/LHCC 99-14, 1999.

4. SUGRA Working Group, S. Abel et al., Report of the sugra working group for run ii of the tevatron, 2000. [hep$\mathrm{ph} / 0003154]$

5. P. Richardson, PhD thesis, University of Oxford, 2000. [hep-ph/0101105]

6. G. Corcella et al., JHEP 01, 010 (2001). [hep-ph/0011363]

7. N. Ghodbane et al. [hep-ph/9909499]

8. S. Ambrosanio et al., in Tevatron Run II SUSY/Higgs workshop, 1998. [hep-ph/0006162]

9. B. Allanach et al., in Tevatron Run II SUSY/Higgs workshop, 1998. [hep-ph/9906224] 
P. Z. Skands: Searching for L-Violating Supersymmetry at the LHC

ATLAS IL-SUSY DISCOVERY POTENTIAL

\begin{tabular}{|c|c|c|c|c|c|c|c|}
\hline \multirow[b]{2}{*}{$\begin{array}{l}\text { SUSY } \\
\text { Point }\end{array}$} & \multicolumn{3}{|c|}{ NETWORK } & \multirow[b]{2}{*}{$\begin{array}{l}\text { SUSY } \\
\text { Point }\end{array}$} & \multicolumn{3}{|c|}{ NETWORK } \\
\hline & $\begin{array}{l}\text { MSSM } \\
P / P_{\text {corr }}\end{array}$ & $\begin{array}{c}\text { LLE } \\
P / P_{\text {corr }}\end{array}$ & $\begin{array}{l}\text { LQD } \\
P / P_{\text {corr }}\end{array}$ & & $\begin{array}{l}\text { MSSM } \\
P / P_{\text {corr }}\end{array}$ & $\begin{array}{c}\text { LLE } \\
P / P_{\text {corr }}\end{array}$ & $\begin{array}{c}\text { LQD } \\
P / P_{\text {corr }}\end{array}$ \\
\hline$P_{2 a}^{\mathrm{LLE}}$ & $24.3 / 16.2$ & $25.5 / 17.1$ & $25.4 / 17.1$ & $P_{2 a}^{\mathrm{LLE}+\mathrm{LQD}}$ & $24.3 / 16.2$ & $25.9 / 17.4$ & $25.8 / 17.4$ \\
\hline$P_{2 b}^{\mathrm{LLE}}$ & $24.5 / 16.3$ & $25.8 / 17.3$ & $25.7 / 17.3$ & $P_{2 b}^{\mathrm{LLE}+\mathrm{LQD}}$ & $24.5 / 16.3$ & $25.9 / 17.4$ & $25.9 / 17.4$ \\
\hline$P_{2 n}^{\mathrm{LLE}}$ & $23.2 / 15.4$ & $24.7 / 16.5$ & $24.3 / 16.3$ & $P_{2 n}^{\mathrm{LLE}+\mathrm{LQD}}$ & $21.4 / 14.0$ & $23.2 / 15.4$ & $24.2 / 16.2$ \\
\hline$P_{7 a}^{\mathrm{LLE}}$ & $0.7 / 0.4$ & $0.7 / 0.4$ & $0.8 / 0.5$ & $P_{7 a}^{\mathrm{LLE}+\mathrm{LQD}}$ & $0.8 / 0.5$ & $0.8 / 0.5$ & $0.9 / 0.5$ \\
\hline$P_{7 b}^{\mathrm{LLE}}$ & $0.8 / 0.4$ & $0.8 / 0.4$ & $0.8 / 0.5$ & $P_{7 b}^{\mathrm{LLE}+\mathrm{LQD}}$ & $0.8 / 0.5$ & $0.8 / 0.5$ & $0.9 / 0.5$ \\
\hline$P_{7 n}^{\mathrm{LLE}}$ & $0.7 / 0.4$ & $0.7 / 0.4$ & $0.8 / 0.5$ & $P_{7 n}^{\mathrm{LLE}+\mathrm{LQD}}$ & $1.0 / 0.6$ & $1.0 / 0.6$ & $1.1 / 0.6$ \\
\hline$P_{9 a}^{\mathrm{LLE}}$ & $191 / 153$ & $315 / 256$ & $218 / 176$ & $P_{9 a}^{\mathrm{LLE}+\mathrm{LQD}}$ & $179 / 143$ & $289 / 234$ & $203 / 164$ \\
\hline$P_{9 b}^{\mathrm{LLE}}$ & $190 / 153$ & $316 / 256$ & $218 / 176$ & $P_{9 b}^{\mathrm{LEE}+\mathrm{LQD}}$ & $178 / 143$ & $291 / 236$ & $204 / 164$ \\
\hline$P_{9 n}^{\mathrm{LLE}}$ & $166 / 133$ & $257 / 208$ & $169 / 135$ & $P_{9 n}^{\mathrm{LLE}+\mathrm{LQD}}$ & $114 / 89.3$ & $132 / 105$ & $111 / 87.0$ \\
\hline$P_{12 a}^{\mathrm{LLE}}$ & $23.4 / 15.5$ & $25.6 / 17.2$ & $25.5 / 17.2$ & $P_{12 a}^{\mathrm{LLE}+\mathrm{LQD}}$ & $20.7 / 13.6$ & $23.8 / 15.8$ & $24.3 / 16.3$ \\
\hline$P_{12 b}^{\mathrm{LLE}}$ & $23.4 / 15.5$ & $25.5 / 17.2$ & $25.5 / 17.2$ & $P_{12 b}^{\mathrm{LLE}+\mathrm{LQD}}$ & $20.8 / 13.6$ & $23.8 / 15.8$ & $24.4 / 16.4$ \\
\hline$P_{12 n}^{\mathrm{LLE}}$ & $21.8 / 14.4$ & $24.2 / 16.2$ & $24.3 / 16.3$ & $P_{12 n}^{\mathrm{LLE}+\mathrm{LQD}}$ & $16.5 / 10.6$ & $18.5 / 12.0$ & $20.8 / 13.7$ \\
\hline$F_{2 a}^{\mathrm{LLE}}$ & $11.3 / 7.0$ & $14.0 / 8.8$ & $13.3 / 8.4$ & $F_{2 a}^{\mathrm{LLE}+\mathrm{LQD}}$ & $9.0 / 5.5$ & $11.9 / 7.4$ & $11.9 / 7.5$ \\
\hline$F_{2 b}^{\mathrm{LLE}}$ & $11.2 / 6.9$ & $13.7 / 8.7$ & $13.1 / 8.2$ & $F_{2 b}^{\mathrm{LLE}+\mathrm{LQD}}$ & $9.0 / 5.5$ & $11.7 / 7.3$ & $11.8 / 7.4$ \\
\hline$F_{2 n}^{\mathrm{LLE}}$ & $9.9 / 6.1$ & $12.4 / 7.8$ & $12.3 / 7.7$ & $F_{2 n}^{\mathrm{LLE}+\mathrm{LQD}}$ & $7.1 / 4.3$ & $8.9 / 5.5$ & $10.4 / 6.4$ \\
\hline$P_{2 a}^{\mathrm{LQD}}$ & $20.9 / 13.7$ & $24.3 / 16.2$ & $24.8 / 16.6$ & $P_{2}^{\mathrm{MSSM}}$ & $10.4 / 6.4$ & $10.2 / 6.3$ & $10.2 / 6.3$ \\
\hline$P_{2 b}^{\mathrm{LQD}}$ & $21.4 / 14.1$ & $24.7 / 16.5$ & $25.3 / 17.0$ & $P_{7}^{\mathrm{MSSM}}$ & $0.2 / 0.1$ & $0.2 / 0.1$ & $0.2 / 0.1$ \\
\hline$P_{2 n}^{\mathrm{LQD}}$ & $21.5 / 14.1$ & $23.3 / 15.5$ & $24.2 / 16.2$ & $P_{9}^{\mathrm{MSSM}}$ & $136 / 108$ & $121 / 95.9$ & $93.5 / 72.8$ \\
\hline$P_{7 a}^{\mathrm{LQD}}$ & $1.0 / 0.6$ & $1.0 / 0.6$ & $1.1 / 0.6$ & $P_{12}^{\mathrm{MSSM}}$ & $16.1 / 10.3$ & $15.5 / 9.9$ & $16.1 / 10.3$ \\
\hline$P_{7 b}^{\mathrm{LQD}}$ & $1.0 / 0.6$ & $1.0 / 0.6$ & $1.1 / 0.7$ & $F_{2}^{\mathrm{MSSM}}$ & $9.4 / 5.8$ & $9.7 / 6.0$ & $10.5 / 6.5$ \\
\hline$P_{7 n}^{\mathrm{LQD}}$ & $1.0 / 0.6$ & $1.0 / 0.6$ & $1.1 / 0.6$ & & & & \\
\hline$P_{9 a}^{\mathrm{LQD}}$ & $116 / 91.6$ & $153 / 122$ & $125 / 99.0$ & & & & \\
\hline$P_{9 b}^{\mathrm{LQD}}$ & $113 / 88.7$ & $151 / 121$ & $123 / 97.6$ & & & & \\
\hline$P_{9 n}^{\mathrm{LQD}}$ & $113 / 88.5$ & $131 / 104$ & $110 / 86.6$ & & & & \\
\hline$P_{12 a}^{\mathrm{LQD}}$ & $15.7 / 10.0$ & $19.2 / 12.5$ & $20.9 / 13.7$ & & & & \\
\hline$P_{12 b}^{\mathrm{LQD}}$ & $15.8 / 10.1$ & $19.4 / 12.6$ & $21.1 / 13.9$ & & & & \\
\hline$P_{12 n}^{\mathrm{LQD}}$ & $16.6 / 10.6$ & $18.6 / 12.1$ & $20.9 / 13.8$ & & & & \\
\hline$F_{2 a}^{\mathrm{LQD}}$ & $7.0 / 4.2$ & $9.5 / 5.9$ & $10.5 / 6.5$ & & & & \\
\hline$F_{2 b}^{\mathrm{LQD}}$ & $7.0 / 4.2$ & $9.4 / 5.8$ & $10.5 / 6.5$ & & & & \\
\hline$F_{2 n}^{\mathrm{LQD}}$ & $6.9 / 4.2$ & $8.8 / 5.4$ & $10.3 / 6.4$ & & & & \\
\hline
\end{tabular}

Table 5. ATLAS discovery potential and corrected discovery potential (see text) for all SUSY scenarios investigated using each of the three networks. The numbers shown correspond to an integrated luminosity of $30 \mathrm{fb}^{-1}$.

10. D0, V. M. Abazov et al., (2001). [hep-ex/0111053]

11. S. Dimopoulos et al., Phys. Rev. D41, 2099 (1990).

12. H. Dreiner, P. Richardson, M. H. Seymour, (2000). [hep$\mathrm{ph} / 0001224]$

13. S. Abdullin et al., in Physics at TeV Colliders, Les Houches, 1999. [hep-ph/0005142]

14. Y. Xi et al., Phys. Rev. D64 (2001). [hep-ph/0107006]

15. J. Ellis et al., Nucl. Phys. B238, 453 (1984).

16. S. Weinberg, Phys. Rev. D 26, 287 (1982).

17. Super-Kamiokande Collaboration, M. Shiozawa et al., Phys. Rev. Lett. 81, 3319 (1998). [hep-ex/9806014]
18. H. Dreiner, in Perspectives on supersymmetry, ed. G. Kane, chap. 20, (World Scientific, 1998). [hep$\mathrm{ph} / 9707435]$

19. G. R. Farrar, P. Fayet, Phys. Lett. B 76, 575 (1978).

20. G. Gilbert, Nucl. Phys. B 328, 159 (1989).

21. L. M. Krauss, F. Wilczek, Phys. Rev. Lett. 62, 1221 (1989).

22. F. Wegner, J. Math. Phys. 12, 2259 (1971).

23. I. Hinchliffe, T. Kaeding, Phys. Rev. D 47, 279 (1993).

24. L. E. Ibáñez, G. G. Ross, Nucl. Phys. B 368, 3 (1992).

25. T. Banks, M. Dine, Phys. Rev. D 45, 1424 (1992). [hepth/9109045] 
26. H. Dreiner, P. Richardson, M. H. Seymour, JHEP 001 (2000). [hep-ph/9912407]

27. T. Sjöstrand, L. Lönnblad, S. Mrenna. [hep-ph/0108264]

28. P. Skands, Master's thesis, Niels Bohr Inst., 2001. [hep$\mathrm{ph} / 0108207]$

29. H. E. Haber, G. L. Kane, Phys. Rep. 117, 76 (1985), Mixing conventions can be found in App. C.

30. J. F. Gunion, H. E. Haber, Nucl. Phys. B 272, 1 (1986), SLAC-PUB-3404.

31. H. Baer, J. Sender, X. Tata, Phys. Rev. D 50, 4517 (1994). [hep-ph/9404342]

32. A. de Roeck, CLIC, Talk given at the CERN EP-TH Faculty Meeting, 2001.

33. B. C. Allanach, A. Dedes, H. K. Dreiner, Phys. Rev. D 60, 075014 (1999). [hep-ph/9906209]

34. H. Dreiner, G. G. Ross, Nucl. Phys. B365, 597 (1991).

35. T. Sjöstrand, Comp. Phys. Comm. 82, 74 (1994).

36. E. Richter-Was et al., ATLAS Internal Note ATL-PHYS98-131, 1998.

37. G. Altarelli, M. Mangano, editors, Proceedings of the Workshop on Standard Model Physics (and more) at the LHC, 2000, CERN 2000-004.

38. J. Bystricky et al., ATLAS Internal Note (1996), DAQNO-054.

39. Y. LeCun et al., in Advances in Neural Information Processing Systems 2, eds. D. S. Touretsky et al., p. 396, (MIT Press, 1990).

40. H. Baer, C. Chen, X. Tata, Phys. Rev. D 55, 1466 (1997). [hep-ph/9608221]

41. B. C. Allanach et al., JHEP 03, 048 (2001). [hep$\mathrm{ph} / 0102173]$

42. B. C. Allanach et al., JHEP 09, 021 (2001). [hep$\mathrm{ph} / 0106304]$ 